\title{
ANÁLISE DO DESEMPENHO FUNCIONAL E PERFIL SÓCIODEMOGRÁFICO DE UMA POPULAÇÃO COM QUEIXA DE LOMBALGIA
}

Carolina Rodrigues Bortolatto ${ }^{1}$, Mileide Cristina Stoco de Oliveira', Fernanda Mayra Fajalle', Elaine Aparecida Lozano da Silva'.

Universidade Estadual Paulista - UNESP. Residente em Fisioterapia, Presidente Prudente, SP. E-mail: ca.bortolatto@hotmail.com

\section{RESUMO}

O objetivo foi avaliar o desempenho funcional e caracterização de indivíduos com queixas de lombalgia de um grupo de exercícios terapêuticos de uma Estratégia de Saúde da Família (ESF). Foi realizado estudo transversal descritivo com 17 indivíduos, de idade média de 59 anos de ambos os sexos. O desempenho funcional foi avaliado pelo Questionário Roland Morris (RM) e a caracterização dos participantes foram a partir da coleta de dados sociodemográficos, antropométricos, localização da dor lombar, intensidade da dor pela Escala Visual Analógica (EVA). No perfil sociodemográfico a renda média foi de 1,64 salário. 0 índice de massa corporal variou de 22,39 a 38,91 $\mathrm{Kg} / \mathrm{m}^{2}$. O escore do RM variou de 9 a 24 pontos de incapacidade funcional. Os resultados da EVA demonstraram que $41,2 \%$ dos indivíduos caracterizaram a dor lombar como intensa. Conclui-se que o comprometimento funcional dos indivíduos avaliados foi grave, causado pela presença de dores na coluna, principalmente, na região lombar.

Palavras-chave: lombalgia, fisioterapia, questionário Roland Morris, ESF e SUS.

\section{ANALYSIS OF FUNCTIONAL PERFORMANCE AND SOCIO-DEMOGRAPHIC PROFILE OF A POPULATION COMPLAINING OF LOW BACK PAIN}

\begin{abstract}
The objective was to evaluate the functional performance and characterization of individuals with low back pain complaints of a group therapeutic exercise a Family Health Strategy (FHS). A descriptive cross-sectional study carried out with a sample of 17 subjects, mean age of 59 years of both genders. Functional performance was assessed by the Roland Morris Questionnaire (RM) and the characterization of participants were from the collection of demographic data, anthropometric, location of low back pain, pain intensity by Visual Analog Scale (VAS). The sociodemographic profile the average income was 1.64 wages. Body mass index ranged from 22.39 the $38.91 \mathrm{~kg} / \mathrm{m} 2$. The RM score ranged 9-24 points of disability. The results showed that the EVA $41.2 \%$ of individuals characterized as severe back pain. It is concluded that the functional impairment of the individuals was severe, caused by the presence of back pain, especially in the lower back.
\end{abstract}

Keywords: low back pain, physical therapy, Roland Morris questionnaire, ESF and SUS.

\section{INTRODUÇÃO}

Atualmente a lombalgia, também conhecida como dor lombar, é uma das alterações musculoesquelética mais comum nas sociedades industrializadas, podendo afetar de $70 \%$ a $80 \%$ da população adulta em algum momento da vida, sendo essa mais prevalente no sexo feminino e em pessoas com idade entre $40 \mathrm{e}$ 80 anos $^{1-3}$.

A lombalgia é definida como uma dor ou desconforto localizado abaixo da última costela e acima da prega glútea inferior, com ou sem irradiação para os membros inferiores, sendo considerada crônica quando a dor persiste por mais de 3 meses $^{2}$. É considerada incapacitante, pois pode provocar desconfortos e limitações no trabalho, nas atividades domésticas e de lazer, tonando-se uma das razões mais comuns para aposentadoria por incapacidade total ou parcial ${ }^{4}$.

A Pesquisa Nacional de Saúde, publicada no ano de 2014, concluiu que $18,5 \%$ da população adulta do Brasil referiu possuir problema crônico na coluna, totalizando cerca de 27 milhões de pessoas. Sendo os problemas na região lombar os mais comuns e englobam $21 \%$ das mulheres e $15 \%$ dos homens ${ }^{5}$.

Dentre as ações de saúde no Brasil, encontramos a Estratégia de Saúde da Família (ESF) que apresenta uma nova abordagem de intervenção do Sistema Único de Saúde (SUS), colocando a família como o foco principal, introduzindo uma nova visão no processo de atenção à saúde. 
Neste sentido, a abordagem realizada na ESF é de busca ativa, com a equipe multiprofissional indo até a população, conhecendo a família e as suas condições, para possível intervenção na prevenção e necessidades do cuidado ${ }^{6}$. Dessa forma, ocorrem mudanças nas práticas da equipe nos mais diferentes espaços, e neste conjunto a Fisioterapia que pela vivência no campo da reabilitação, tem necessidade de se adequar a essa nova realidade ${ }^{7}$, com ações voltadas para prevenção e atenção básica à saúde.

A fisioterapia compõe o núcleo de profissionais que dão suporte para as ESF, composto por outros profissionais da área da saúde. Neste contexto, foi verificado pela equipe de fisioterapia grande parte da população participante do Grupo de Exercícios Terapêuticos para dores crônicas relatavam dores na coluna, principalmente, na região lombar. Por isso, o objetivo deste estudo foi realizar uma avaliação sobre o desempenho funcional e caracterização destes indivíduos com queixas de lombalgia.

\section{MÉTODOS}

Foi realizado um estudo transversal descritivo, com indivíduos adultos de ambos os sexos, participam do Grupo de Exercícios Terapêuticos para dores crônicas em uma ESF do município de Presidente Prudente e que relataram queixas de lombalgia. Todos os indivíduos foram informados sobre os procedimentos do estudo e o trabalho foi aprovado pelo comitê de ética CAAE: 05466712.4.0000.5402.

O estudo foi composto pela coleta dos dados sociodemográficos, antropométricos, localização da dor lombar, intensidade da dor pela Escala Visual Analógica (EVA) e aplicação do Questionário Roland Morris (RM).

Os dados sociodemográficos e antropométricos foram coletados a partir de uma ficha própria. Os dados sociodemográficos continham o nome, a idade (em anos completos), o sexo, a renda (em salários), a situação conjugal e a situação ocupacional (nas categorias trabalhando e não trabalhando); já os dados antropométricos mediram o peso (em $\mathrm{Kg}$ ) e a altura (em metros), o Índice de Massa Corpórea (em $\mathrm{Kg} / \mathrm{m}^{2}$ ) e a circunferência abdominal (em centímetros).

A mensuração da circunferência abdominal ocorreu por meio de uma fita métrica comum e utilizada a medida do ponto médio entre a crista ilíaca e o rebordo costal inferior ${ }^{8}$. Os valores do IMC e da circunferência abdominal serão classificados de acordo com a III Diretrizes Brasileiras de Obesidade ${ }^{8}$.

A localização da dor lombar foi determinada ao se apresentar ao indivíduo uma figura esquemática de uma pessoa em posição ereta, supina e dorsal com as regiões cervical, torácica e lombar pintadas em cores diferentes (Figura 1) em que o indivíduo deveria apontar a região com a cor vermelha ${ }^{9}$.

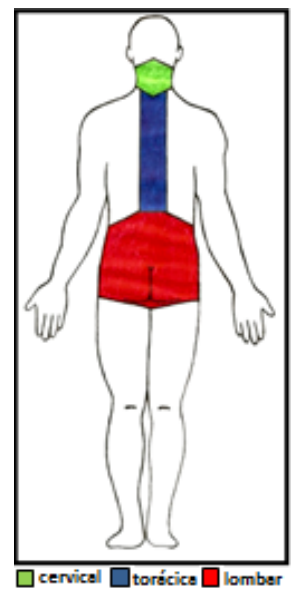

Figura 1. Pessoa na posição ereta, supina e dorsal com as regiões cervical, torácica e lombar pintadas.

A Escala Visual Analógica foi utilizada para quantificar a intensidade e a percepção do indivíduo sobre sua dor, sendo graduada de acordo com pontuações ordinais: nenhuma dor (0), dor leve (1 a2), moderada (3 a 5) e intensa (6 a 10).

O Questionário Roland Morris (RM) foi utilizado para avaliar o desempenho funcional dos participantes. Esse questionário é constituído de 24 itens que exemplificam consequências funcionais decorrentes da lombalgia. O Roland Morris foi traduzido para o português e adaptado para a população brasileira. Este questionário tem como ponto de corte o escore "14", ou seja, os indivíduos avaliados com um escore igual ou maior que "14" são classificados como incapacitados funcionalmente. Além disso, esse instrumento foi escolhido por ser de simples apresentação e ter um sistema de escore padronizado $^{10}$.

A aplicação do RM ocorreu em local reservado, sendo as perguntas do questionário 
lidas pelo examinador, e o escore foi calculado em relação ao número de respostas afirmativas do paciente. Dessa forma, quanto maior o escore, maior a incapacidade do indivíduo. Os participantes foram orientados a responder às questões levando-se em conta a sua condição no momento da apresentação do teste.

\section{RESULTADOS}

A população estudada foi composta por 16 mulheres e 1 homem, com idade de 59,1 $\pm 9,8$ anos. A renda média foi de $1,64 \pm 0,84$ salário. 0 peso dos participantes variou de 54,5 a $93,5 \mathrm{~kg}$ (média de $74,4 \pm 11,8$ ), a altura variou de 1,57 a $1,65 \mathrm{~m}$ e o IMC variou de 22,39 a $38,91 \mathrm{Kg} / \mathrm{m}^{2}$, como mostrado na Tabela 1.

Tabela 1. Características sociodemográficas e de saúde dos indivíduos.

\begin{tabular}{|c|c|c|}
\hline Variáveis & $\mathbf{n}$ & $\%$ \\
\hline \multicolumn{3}{|l|}{ Sexo } \\
\hline Feminino & 16 & 94,1 \\
\hline Masculino & 1 & 5,9 \\
\hline \multicolumn{3}{|l|}{ Idade } \\
\hline 20-39 & 1 & 5,9 \\
\hline $40-59$ & 7 & 41,1 \\
\hline $60-79$ & 8 & 47,1 \\
\hline$\geq 80$ & 1 & 5,9 \\
\hline \multicolumn{3}{|l|}{ Situação conjugal (confirmar) } \\
\hline Casado & 11 & 64,8 \\
\hline Separado & 1 & 5,9 \\
\hline Solteiro & 2 & 11,7 \\
\hline Viúvo & 3 & 17,6 \\
\hline \multicolumn{3}{|l|}{ Situação ocupacional } \\
\hline Trabalhando & 3 & 17,6 \\
\hline Não trabalhando & 14 & 82,4 \\
\hline \multicolumn{3}{|l|}{ Renda familiar } \\
\hline 1 salário & 10 & 58,8 \\
\hline 2 salários & 5 & 29,5 \\
\hline$\geq 3$ salários & 2 & 11,7 \\
\hline \multicolumn{3}{|l|}{$\mathrm{IMC}\left(\mathrm{Kg} / \mathrm{m}^{2}\right)$} \\
\hline Normal $(18,5$ a 24,9$)$ & 1 & 5,9 \\
\hline Pré-obeso (25 a 29,9) & 7 & 41,2 \\
\hline Obesidade I (30 a 34,9) & 8 & 47,0 \\
\hline Obesidade II $(35$ a 39,9$)$ & 1 & 5,9 \\
\hline \multicolumn{3}{|l|}{ Circunferência abdominal $(\mathrm{cm})$} \\
\hline Homem ( $\geq 102)$ & 1 & 100,0 \\
\hline Mulheres ( $\geq 88)$ & 14 & 87,5 \\
\hline
\end{tabular}

No que se refere à localização da dor na coluna ocorreu o predomínio da região lombar, seguido de dor lombar e cervical, e as regiões lombar e torácica, apresentado na Tabela 2. O escore do RM variou de 9 a 24 pontos de incapacidade funcional (média 14,83 \pm 4,53 pontos).

Tabela 2. Localização da dor na coluna.

\begin{tabular}{lcc}
\hline Características & $\mathbf{n}$ & $\mathbf{\%}$ \\
\hline Lombar & 8 & 47,0 \\
Lombar + cervical & 4 & 23,5 \\
Lombar + torácica & 3 & 17,7 \\
$\begin{array}{l}\text { Lombar + torácica + } \\
\text { cervical }\end{array}$ & 2 & 11,8 \\
\hline
\end{tabular}

Os resultados encontrados a partir da EVA demonstraram que $41,2 \%$ dos indivíduos caracterizaram a dor lombar como intensa, com média de 8,1 $( \pm 0,8)$ pontos (Tabela 3$)$.

Tabela 3. Caracterização da dor de indivíduos com lombalgia de acordo com a Escala Visual Analógica.

\begin{tabular}{lccc}
\hline EVA & $\mathbf{n}$ & $\mathbf{\%}$ & Média \pm DP \\
\hline Sem dor (0) & 1 & 5,9 & - \\
Leve (1 a 2) & 3 & 17,6 & $1,3 \pm 0,5$ \\
Moderada (3 a 5) & 6 & 35,3 & $3,8 \pm 0,7$ \\
Intensa (6 a 10) & 7 & 41,2 & $8,1 \pm 0,8$ \\
\hline
\end{tabular}

O questionário RM apresentou variação do escore de 9 a 24 pontos de incapacidade funcional, e média de $14,83 \pm 4,53$ pontos. $\mathrm{Na}$ mensuração da incapacidade funcional foi observado que $58,8 \%$ dos indivíduos foram classificados como portadores de incapacidade funcional devido à lombalgia (Tabela 4).

Tabela 4. Incapacidade funcional de indivíduos com dor lombar por meio do Questionário Rolland-Morris.

\begin{tabular}{lcc}
\hline Pontuação & $\mathbf{n}$ & $\%$ \\
\hline 0 a 6 pontos & 0 & 0 \\
7 a 13 pontos & 7 & 41,2 \\
14 ou mais pontos & 10 & 58,8 \\
\hline
\end{tabular}

\section{DISCUSSÃO}

O estudo realizado por Azevedo et al. ${ }^{11}$ obteve como resultado a prevalência de dores lombares em indivíduos com sobrepeso, quando comparado ao indivíduo com peso normal, corroborando com o presente estudo.

Os participantes do presente estudo apresentaram valores IMC e circunferência 
abdominal acima dos valores preditos como normalidade, com média de $29,90 \pm 3,96 \mathrm{Kg} / \mathrm{m}^{2}$ e para circunferência abdominal de $102 \mathrm{~cm}$ para homens e $94,91 \mathrm{~cm}$ para mulheres. Sabe-se que o excesso de peso ocasiona sobrecarga em toda a coluna, e principalmente na região lombar devido ao aumento da circunferência abdominal que gera alterações na curvatura da lombar ${ }^{12,13}$.

Atualmente a prevalência de dor na região lombar é encontrada com frequência na população, dado encontrado no presente estudo, porém a dor na lombar pode ocorrer associada a outra região da coluna, assim $47,05 \%$ dos participantes relataram sentir dores na região lombar, $23,54 \%$ região lombar e cervical, $17,65 \%$ região lombar e torácica e $11,76 \%$ nas regiões lombar, torácica e cervical. No estudo composto por 972 indivíduos ocorreu a predominância de dores na região lombar, seguida da região torácica e cervical ${ }^{14}$.

Com relação à incapacidade funcional nenhum indivíduo apresentou escore de 0 a 6 pontos no questionário RM, $41,17 \%$ apresentou escore de 7 a 13 pontos e 58,8 \% com escore de 14 ou mais pontos. Já Mascarenhas e Santos ${ }^{15}$, ao avaliar a incapacidade funcional de indivíduos com lombalgia por meio do questionário RM verificaram a predominância de 7 a 13 pontos.

Diversos estudos mostraram que após os protocolos de exercícios específicos para a musculatura profunda do tronco (MPT) houve melhora significativa dos níveis de flexibilidade e funcionalidade, demonstrando a redução da dor e incapacidade, além de diminuir as taxas de recorrência após um episódio de dor aguda ${ }^{16-19}$.

O presente estudo possibilitou constatar a necessidade de inclusão de exercícios de estabilização segmentar que consistem na contração da MPT, transverso do abdômen e multífidos no Grupo de Exercícios Terapêuticos, pois a prática regular de exercícios utilizando halteres e caneleiras não está produzindo efeito de fortalecimento da musculatura profunda do tronco.

\section{CONCLUSÃO}

Pode-se concluir que houve a predominância de indivíduos do sexo feminino, com idade de 60 á 79 anos, casados, não trabalhando e com renda de um salário mínimo, incapacidade funcional grave e presença de dores na região lombar.
A realização de exercícios terapêuticos é fundamental para auxiliar na diminuição do quadro incapacitante de pacientes com dor lombar. E proporcionarem aos indivíduos melhores condições de vida, principalmente no âmbito socioeconômico, o que acaba sendo afetada devido às limitações do quadro de dor.

\section{CONFLITO DE INTERESSE}

Os autores declaram não haver qualquer potencial de conflito de interesse que possa interferir na imparcialidade deste trabalho científico.

\section{REFERÊNCIAS}

1. Hoy DG, Bain C, Williams G, March L, Brooks P, Blyth $F$, Woolf A, Vos T, Buchbinder R. A systematic review of the global prevalence of low back pain. Arthritis Rheumatol. 2012;64(6):2028-37. DOI: http://dx.doi.org/10.1002/art.34347

2. Riberto M, Chiappetta LM, Lopes KAT, Battistella LR. A transversal multicenter study assessing functioning, disability and environmental factors with the comprehensive ICF core set for low back pain in Brazil. Eur J Phys Rehab Med. 2014;50:153-60.

3. Associação Brasileira de Medicina Física e Reabilitação. Lombalgia inespecífica crônica: reabilitação. Rev Assoc Med Bras. 2013;59(6):536-53. http://dx.doi.org/10.1016/j.ramb.2013.10.003

4. World Health Organization. Quantifying selected major risks to health. World Health Report - Chapter four. 2002.

5. Instituto Brasileiro de Geografia e Estatística. Pesquisa Nacional de Saúde 2013: percepção do estado de saúde, estilos de vida e doenças crônicas: Brasil, grandes regiões e unidades da federação. Rio de Janeiro: Instituto Brasileiro de Geografia e Estatística; 2014.

6. Rosa WAG, Labate RC. Programa saúde da família: a construção de um novo modelo de assistência. Rev Latino-Am Enferm. 2005;13(6):1027-34. DOI: http://dx.doi.org/10.1590/S0104-

\section{6}

7. Souza MC, Bonfim AS, Souza JN, Franco, TB. Fisioterapia e Núcleo de Apoio à Saúde da Família: conhecimento, ferramentas e desafios. Mundo saúde. 2013;2(37):176-84.

8. Associação Brasileira para o Estudo da Obesidade e da Síndrome Metabólica. III Diretrizes Brasileiras de Obesidade. 2009/2010.

9. Meucci RD, Fassa AG, Paniz VMV, Silva MC, Wegman DH. Increase of chronic low back pain prevalence in a medium-sized city of southern Brazil. BMC Musculo skelet Disord. 2013;14:155. DOI: http://dx.doi.org/10.1186/1471-2474-14-155 
10. Nusbaum L, Natour J, Ferraz MB, Goldenberg J. Translation, adaptation and validation of the rolandmorris questionnaire - Brazil. Braz J Med Biol Res. 2001;34(2):203-10. DOI: http://dx.doi.org/10.1590/S0100-

\section{X2001000200007}

11. Azevedo JVS, Silva JRL, Ribeiro DCL. Relação entre lombalgia e sobrepeso em praticantes de atividade física. ConScientiae Saúde. 2008;7(4):471-475.

12. Contri DE, Pereira TFG, Canelhas MRL. A obesidade e lombalgia- proposta de métodos alternativos para diminuição da massa corporal e alívio da dor: relato de caso. ConScientiae Saúde, 2009;8(3):509-14. DOI: http://dx.doi.org/10.5585/conssaude.v8i3.1775

13. Almeida ICGB, Sá KN, Silva M, Baptista A, Matos $M A$, Lessa I. Prevalência de dor lombar crônica na população da cidade de Salvador. Rev Bras Ortop. 2008;43(3):96-102. DOI:

http://dx.doi.org/10.1590/s010236162008000200007

14. Ferreira GD, Silva MC, Rombaldi AJ, Wrege ED, Siqueira FV, Hallal PC. Prevalência de dor nas costas e fatores associados em adultos do Sul do Brasil: estudo de base populacional. Rev Bras Fisioter. 2011;15(1):316.

15. Mascarenhas CHM, Santos LS. Avaliação da dor e da capacidade funcional em indivíduos com lombalgia crônica. J Health Sci Inst. 2011;29(3):205-8.

16. Pereira NT, Ferreira LAB, Pereira WM. Efetividade de exercícios de estabilização segmentar sobre a dor lombar crônica mecânico-postural. Fisioter Mov. 2010;23(4):605-14.

DOI:

http://dx.doi.org/10.1590/s0103-

51502010000400011

17. França FJR, Burke TN, Claret DC, Marques AP. Estabilização segmentar da coluna lombar nas lombalgias: uma revisão bibliográfica e um programa de exercícios. Fisiotera Pesq. 2008;15(2):2006. DOI: http://dx.doi.org/10.1590/S1809-

29502008000200015

18. Isokinetic performance, functionality, and pain level before and after lumbar and pelvic estabilization exercise in individuals with chronic low back pain. Fisioter Mov. 2014;27(3):447-55. DOI:

http://dx.doi.org/10.1590/0103-5150.027.003.AO16 19. Bordiak FC, Silva EB. Eletroestimulação e core training sobre dor e arco de movimento na lombalgia. Fisioter Mov. 2012;25(4):759-66. DOI: http://dx.doi.org/10.1590/S0103-

$\underline{51502012000400008}$

Recebido para publicação em 28/08/2015

Revisado em 02/09/2015

Aceito em 05/09/2015 